Vol. 1, No. 1, 2019

https://doi.org/10.23939/cds2019.01.027

УДК 004.451(86); УДК 512.8

P. Kosobutskyy

Lviv Polytechnic National University

\title{
ON THE UNIVERSAL REGULARITY OF THE NUMBERS OF GENERALIZED RECURRENCE SEQUENCE AND SOLUTIONS TO ITS CHARACTERISTIC EQUATION OF SECOND ORDER
}

(C) Kosobutskyy P., 2019

In this work shows that the classical oscillations of the ratio of neighboring members of the Fibonacci sequences are valid for arbitrary directions on the plane of the phase coordinates, approaching, to a maximum, the solutions to the characteristic quadratic equation at a given point. The values of the solutions to the characteristic equation along the satellites are asymptotically close to their integer values of the corresponding root lines.

Keywords: Golden ratio, Phidias number, the quadratic equation, second order recursive sequence.

\section{Introduction}

Many processes and phenomena are modeled by the separation of characteristics systems into parts in certain ratios. In electrical and dynamic systems, the structuring of systems is implemented by serial and parallel, multilayer coatings, and the like. In such systems, mathematical models are justified on the basis of the laws of conservation of energy, charge, and light flux [1,2].

A well-known approach is that in addition to conservation laws, the system structuring model is complemented by a target limitation - the separation of the additive parameter $L$ by the golden ratio (GP). Its essence is that the point of one-dimensional separation with the coordinate into two unequal parts satisfies the proportion

$$
\frac{L}{x}=\frac{x}{L-x}
$$

or in dimensionless units

$$
\begin{aligned}
& \Phi=\frac{L}{x} \text { і } \phi=\frac{x}{L}, \\
\Phi=\frac{1}{\Phi-1} & \text { чи } \quad \frac{1}{\phi}=\frac{\phi}{1-\phi} .
\end{aligned}
$$

with quantitative characteristics

$$
\left\{\begin{array} { l } 
{ \Phi ^ { 2 } - \Phi - 1 = 0 , } \\
{ \phi ^ { 2 } + \phi - 1 = 0 , }
\end{array} ( a ) \Rightarrow \left\{\begin{array}{l}
\Phi_{ \pm}=\frac{1}{2}(1 \pm \sqrt{5})=\left\{\begin{array}{l}
\Phi_{+}=+1.618 \ldots, \\
\Phi_{-}=-0.618 \ldots,
\end{array} \quad(b)\right. \\
\phi_{ \pm}=-\frac{1}{2}(1 \pm \sqrt{5})=\left\{\begin{array}{l}
\phi_{+}=+0.618 \ldots, \\
\phi_{-}=-1.618 \ldots
\end{array}\right.
\end{array}\right.\right.
$$

in the form of known numbers of Phidias and [1]. These numbers are the roots of the quadratic equation

$$
\frac{L}{x}=\frac{x}{L-x} \Rightarrow L^{2}=x^{2}+(\sqrt{L x})^{2} \Rightarrow\left\{\begin{array}{l}
\Phi^{2}-\Phi-1=0, \\
\phi^{2}+\phi-1=0
\end{array}\right.
$$

and according to the theorem of Vieta satisfy the so-called "golden" combinations

$$
\Phi-\phi=1, \Phi \cdot \phi=1 \text { i } \frac{1}{\phi}-\frac{1}{\Phi}=1,
$$


and representation in the form of chain fractions $\left\{\begin{array}{l}\Phi=1+\frac{1}{1+\frac{1}{1+\ldots}}, \\ \phi=1-\frac{1}{1-\frac{1}{1-\ldots}} .\end{array}\right.$ та $\left\{\begin{array}{l}\Phi=\sqrt{1+\sqrt{1+\sqrt{1+\ldots}}}, \\ \phi=\sqrt{1-\sqrt{1-\sqrt{1-\ldots}}}\end{array}\right.$

and patterns of raising to the degree of:

$$
\left\{\begin{array} { l } 
{ \Phi ^ { 2 } - \Phi - 1 = 0 , } \\
{ \phi ^ { 2 } + \phi - 1 = 0 , }
\end{array} \Rightarrow \left\{\begin{array} { c } 
{ \Phi ^ { 3 } - \Phi ^ { 2 } = \Phi , } \\
{ \phi ^ { 3 } + \phi ^ { 2 } = \phi , }
\end{array} \cdots \left\{\begin{array}{c}
\Phi^{n}-\Phi^{n-1}=\Phi^{n-2} \\
\phi^{n}+\phi^{n-1}=\phi^{n-2},
\end{array}, \Rightarrow\left\{\begin{array}{l}
\Phi^{n}=\Phi^{n-1}+\Phi^{n-2}, \\
\phi^{n}=-\phi^{n-1}+\phi^{n-2}
\end{array}\right.\right.\right.\right.
$$

The basis of "golden proportion" is based on methods of additivity and multiplicity. Indeed, when the numerator of each fraction is replaced by the denominator of the previous fraction, and the denominator by the sum of the numerator and denominator of the same fraction, then we obtain:

$$
\tau(1)=\frac{1}{1}, \tau(2)=\frac{1}{2}, \tau(3)=\frac{2}{3}, \tau(4)=\frac{3}{5}, \ldots \Rightarrow \tau(n+1)=\frac{1}{1+\tau(n)} .
$$

So if $\tau(n)=\frac{m}{k}$, so

$$
\tau(n+1)=\frac{k}{k+m}=\frac{1}{1+m / k}=\frac{1}{1+\tau(n)} .
$$

and you can write a sequence of numbers:

$$
\frac{1}{1}, \frac{1}{2}, \frac{2}{3}, \frac{3}{5}, \frac{5}{8}, \ldots
$$

Sequence (10) is interesting in that in each number of the numerator and denominator, starting from the third, is represented as the sum of the two preceding adjacencies - the Fibonacci numbers.

If you take three consecutive terms $u(n), u(n+1), u(n+2)$, then the ratio

$$
\frac{u(n)}{u(n+1)}, \frac{u(n+1)}{u(n+2)}, \frac{u(n+2)}{u(n+3)}, \ldots
$$

are adjacent fractions of the number $\tau$ for which recurrence is established on the basis of (9)

$$
\tau(n+1)+\tau(n+1) \tau(n)=1
$$

Therefore, if the Phidias number $\Phi=\frac{1+\sqrt{5}}{2}$ is represented as a chain fraction $\Phi=1+\frac{1}{1+\frac{1}{1+\ldots}}$ approximated by a suitable fraction $\frac{p}{q}$, then we obtain a sequence consisting of relations of adjacent Fibonacci numbers $\frac{1}{1}, \frac{1}{2}, \frac{2}{3}, \frac{3}{5}, \frac{5}{8}, \ldots$.

On the other hand, the gold section method is used to create optimization algorithms, which implements the geometry of separation over the GR uncertainty interval. Although such an algorithm has a fairly high computational efficiency, it is not optimal for a given number of calculations of the objective function. Indeed, with the symmetric arrangement of the points of calculation of the values of the objective function, the GR method can be combined with the known dichotomy method and an optimal algorithm can be constructed in which the length of the last uncertainty interval is equal to:

$$
L_{N}=\frac{1}{F_{N}}\left(1+F_{N-2} \cdot \varepsilon\right)
$$


Therefore, at the boundary $\varepsilon \rightarrow 0$, the lower bound is equal to the smallest uncertainty interval for a given number of calculations of the objective function. In formula (13) there are Fibonacci numbers $F_{N}$ that appear when solving the second order recurrence relation [3-6]

$$
F(n)=\left\{\begin{array}{c}
F(0)=0 \\
F(1)=1 \\
F(n-1)+F(n-2), n \geq 2
\end{array}\right.
$$

the characteristic equation of which is also the quadratic equation of type (7).

Therefore is known [4] that the roots of equation (1) $\phi, \Phi$ (Phidias numbers) are the boundaries of relations

$$
\lim _{n \rightarrow \infty} \frac{F(n)}{F(n+1)}=\phi \quad \text { or } \quad \lim _{n \rightarrow \infty} \frac{F(n+1)}{F(n)}=\Phi,
$$

neighboring numbers of second-order recurrence sequences

$$
F(n+2)=F(n+1)+F(n) .
$$

This paper shows that on a plane of phase coordinates $(p, q)$ along parallel directions to root lines (satellites)

$$
-\mathrm{K} p+q+\mathrm{K}^{2}=0, \quad \mathrm{~K}=1,2,3, \ldots,
$$

the boundaries of the fluctuations of relations

$$
\lim _{n \rightarrow \infty} \frac{F(p, q, n+2)}{F(p, q, n+1)} \quad \text { and } \quad \lim _{n \rightarrow \infty} \frac{F(p, q, n)}{F(p, q, n+1)}
$$

neighboring elements of a generalized sequence $\{F(p, q, n)\}$ (in the notation of Horadam [7, 8])

$$
F(p, q, n+2)=p F(p, q, n+1)+q F(p, q, n), \quad n \geq 0,
$$

are equal to the value of the roots $x_{ \pm}(p, q)$ of the general characteristic equation

$$
x(p, q)^{2}=p \cdot x(p, q)+q
$$

of sequences (19).

\section{Results and their discussion}

Each quadratic function (20) corresponds to a point with coordinates $(p, q)$ on a plane of phase coordinates $(p, q)$, where the solutions are valid given $p \geq 0$ (second) and $p\langle 0$ (third) quadrants. In the first $p \geq 0, q\rangle 0)$ and the fourth $p\langle 0, q\rangle 0$ quadrants, there are areas with a negative discriminant $D$, where the solutions are imaginary. The true solutions are

$$
x_{ \pm}(p, q)=\frac{p}{2}(1 \pm D), D=\sqrt{p^{2}-4 q} .
$$

In the points $(p, q)$, where the discriminator $D$ greater than zero is not an exact square, the solutions (21) are quadratically irrational. The best known of them is at the point $p= \pm 1, q=-1$, where $D=\sqrt{5}$ and the regularity is realized (1). The discriminant is the exact square along the directions of the root lines (17), so along them the solutions (21) are integers.

The problem of finding a general term of a numerical sequence satisfying (19) is equivalent to the problem of solving the linear differential equation of the second order [7]:

$$
F(p, q, n+2)-p \cdot F(p, q, n+1)-q \cdot F(p, q, n)=0
$$

with the characteristic equation of (20). Therefore, the general solution to equation (22) depends on the solution of the characteristic equation (20). Given a discriminant $D \neq 0$, the roots (20) set two solutions $x_{ \pm}(p, q)$ and 
the geometric progression $\left\{F_{n}\right\}=c_{ \pm} \cdot x_{ \pm}(p, q)^{n}$ will satisfy (9) only given the equation (20) is a characteristic one of sequence (22). Then for a fixed pair $F(0), F(1)$, there is always one pair of numbers $c_{ \pm}$and

$$
F(n)=c_{+}\left(x_{+}(p, q)\right)^{n}+c_{-}\left(x_{-}(p, q)\right)^{n} .
$$

Taking into account the initial conditions

$$
\left\{\begin{array}{c}
c_{+} x_{+}(0)+c_{-} x_{-}(0)=F(0), \\
c_{+} x_{+}(1)+c_{-} x_{-}(1)=F(1),
\end{array} \Rightarrow \begin{array}{l}
c_{+}=\frac{F(1)-F(0) x_{-}(p, q)}{x_{+}(p, q)-x_{-}(p, q)}, \\
c_{-}=-\frac{F(1)-F(0) x_{+}(p, q)}{x_{+}(p, q)-x_{-}(p, q)} .
\end{array}\right.
$$

we get that the formula for calculating an arbitrary element (19):

$$
F(n)=\frac{\left(x_{+}(p, q)\right)^{n-1} \cdot\left(F(1)-F(0) \cdot x_{-}(p, q)\right)-\left(x_{-}(p, q)\right)^{n-1} \cdot\left(F(1)-F(0) \cdot x_{+}(p, q)\right)}{x_{+}(p, q)-x_{-}(p, q)} .
$$

Thus, the ratio is

$$
\begin{aligned}
& \frac{F(p, q, n+2)}{F(p, q, n+1)}=\frac{\left(x_{+}(p, q)\right)^{n+1} \cdot\left(F(1)-F(0) \cdot x_{-}(p, q)\right)-\left(x_{-}(p, q)\right)^{n+1} \cdot\left(F(1)-F(0) \cdot x_{+}(p, q)\right)}{\left(x_{+}(p, q)\right)^{n} \cdot\left(F(1)-F(0) \cdot x_{-}(p, q)\right)-\left(x_{-}(p, q)\right)^{n} \cdot\left(F(1)-F(0) \cdot x_{+}(p, q)\right)}= \\
& =x_{+}(p, q) \frac{1-\left(\frac{x_{-}(p, q)}{x_{+}(p, q)}\right)^{n+1} \cdot \Xi(p, q)}{1-\left(\frac{x_{-}(p, q)}{x_{+}(p, q)}\right)^{n} \cdot \Xi(p, q)}, \quad \Xi(p, q)=\frac{\left(F(1)-F(0) \cdot x_{+}(p, q)\right)}{\left(F(1)-F(0) \cdot x_{-}(p, q)\right)} .
\end{aligned}
$$

Since given the values of fixed numbers $F(0)=0, F(1)=1$, a function $\Xi(p, q)=1$, the ratio (26) equals

$$
\frac{F(p, q, n+2)}{F(p, q, n+1)}=x_{+}(p, q) \frac{1-\left(\frac{x_{-}(p, q)}{x_{+}(p, q)}\right)^{n+1}}{1-\left(\frac{x_{-}(p, q)}{x_{+}(p, q)}\right)^{n}} .
$$

Taking into account inequality $\left|x_{-}(p, q)\right|\left\langle\left|x_{+}(p, q)\right|\right.$, то $\lim _{s \rightarrow \infty}\left(\frac{x_{-}(p, q)}{x_{+}(p, q)}\right)^{s} \rightarrow 0$, we arrive at the conclusion that the boundary of relations (26) or (27)

$$
\lim _{n \rightarrow \infty} \frac{F(p, q, n+2)}{F(p, q, n+1)}=x_{+}(p, q)
$$

is the root of the characteristic equation (20) at the point with the given phase coordinates $(p, q)$, which is consistent with [10] and with a one-to-one correspondence between irrational numbers and boundless chain fractions [12].

To confirm this conclusion, Fig. 1 shows graphical dependences of numerical calculations of values (28) along the phase directions of the first left $\left|q_{l}\right|=|p+2|$ (Fig. 1(a)) and the first right $\left|q_{r}\right|=|p|-1$ (Fig. 1 (b)) satellites of the first $\mathrm{K}=1$ root line. We see that the regularity (28) is valid for arbitrary values of phase coordinates. Similar regularities are valid for the ratio $\lim _{n \rightarrow \infty} \frac{F(p, q, n+2)}{F(p, q, n+1)}$ along the direction of the root line, where the solutions to equation (20) take the integer values, and for higher orders of root lines and their satellites along all directions on the plane of phase coordinates $(p, q)$ in the second and third quadrants. 
The boundary values of the ratio $\lim _{n \rightarrow \infty} \frac{F(p, q, n+2)}{F(p, q, n+1)}$ are different, since the boundaries (21) are different at different phase points $(p, q)$. As follows from Fig. 2(a), given the directions of the root lines $\mathrm{K}=1$ and $\mathrm{K}=2$ and their right $\left(\begin{array}{c}\mathrm{K}=1: q=(p+1)-1 \\ \mathrm{~K}=2: q=(2 p+4)-1\end{array}\right)$ and left $\left(\begin{array}{c}\mathrm{K}=1: q=(p+1)+1 \\ \mathrm{~K}=2: q=(2 p+4)+1\end{array}\right)$ satellites, as shown in Fig. 2(b), with increasing coefficients $p, q$, the values of the solutions $x_{+}(p, q)$ asymptotically go towards the integer values of the corresponding lines along the $\operatorname{root}\left(\begin{array}{c}\mathrm{K}=1: q=(p+1) \\ \mathrm{K}=2: q=(2 p+4)\end{array}\right)$. This conclusion is valid for the directions of all root lines.

The formulated conclusion is confirmed by elementary transformations:

$$
\begin{gathered}
\frac{p U(p, q, n)+q U(p, q, n-1)}{p U(p, q, n-1)+q U(p, q, n-2)}=\frac{p \frac{U(p, q, n)}{U(p, q, n-1)}+q}{p+q \frac{U(p, q, n-2)}{U(p, q, n-1)}} . \text { If } \lim _{n \rightarrow \infty} \frac{U(p, q, n)}{U(p, q, n-1)}= \\
=\lim _{n \rightarrow \infty} \frac{U(p, q, n-2)}{U(p, q, n-1)}=x(p, q), \text { then } \frac{p U(p, q, n)+q U(p, q, n-1)}{p U(p, q, n-1)+q U(p, q, n-2)}=\frac{p x(p, q)+q}{p+q \frac{1}{x(p, q)}}= \\
=x(p, q) \frac{p x(p, q)+q}{p x(p, q)+q}=x(p, q) .
\end{gathered}
$$

Thus, under the initial condition $\frac{F(p, q, n)}{F(p, q, n-1)}=\Phi$ the relation $\frac{p F(p, q, n)+q F(p, q, n-1)}{p F(p, q, n-1)+q F(p, q, n-2)}$ $[9,11-12]$ is equivalent to the relation $\frac{F(p, q, n)}{F(p, q, n-1)}$, so as

$$
\text { i.e. } a=p=1, \quad b=q=1 \text {. }
$$

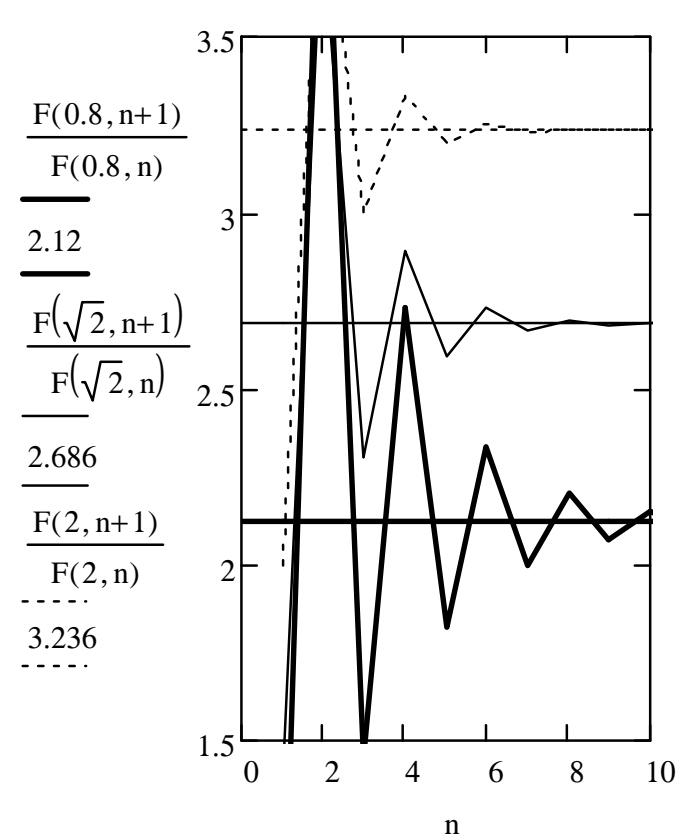

$a$

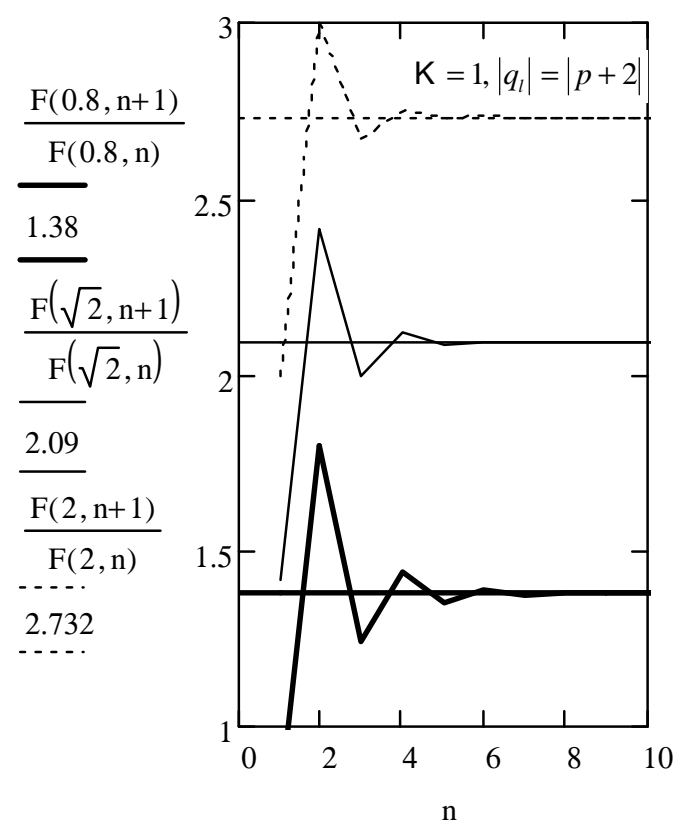

$b$

Fig. 1. The oscillations of the ratio $\frac{F(n+1)}{F(n)}$ along the phase directions of the first left $\left|q_{l}\right|=|p+2|$ (a) and right $\left|q_{r}\right|=|p|(b)$ satellites of the first $\mathrm{K}=1$ root line 

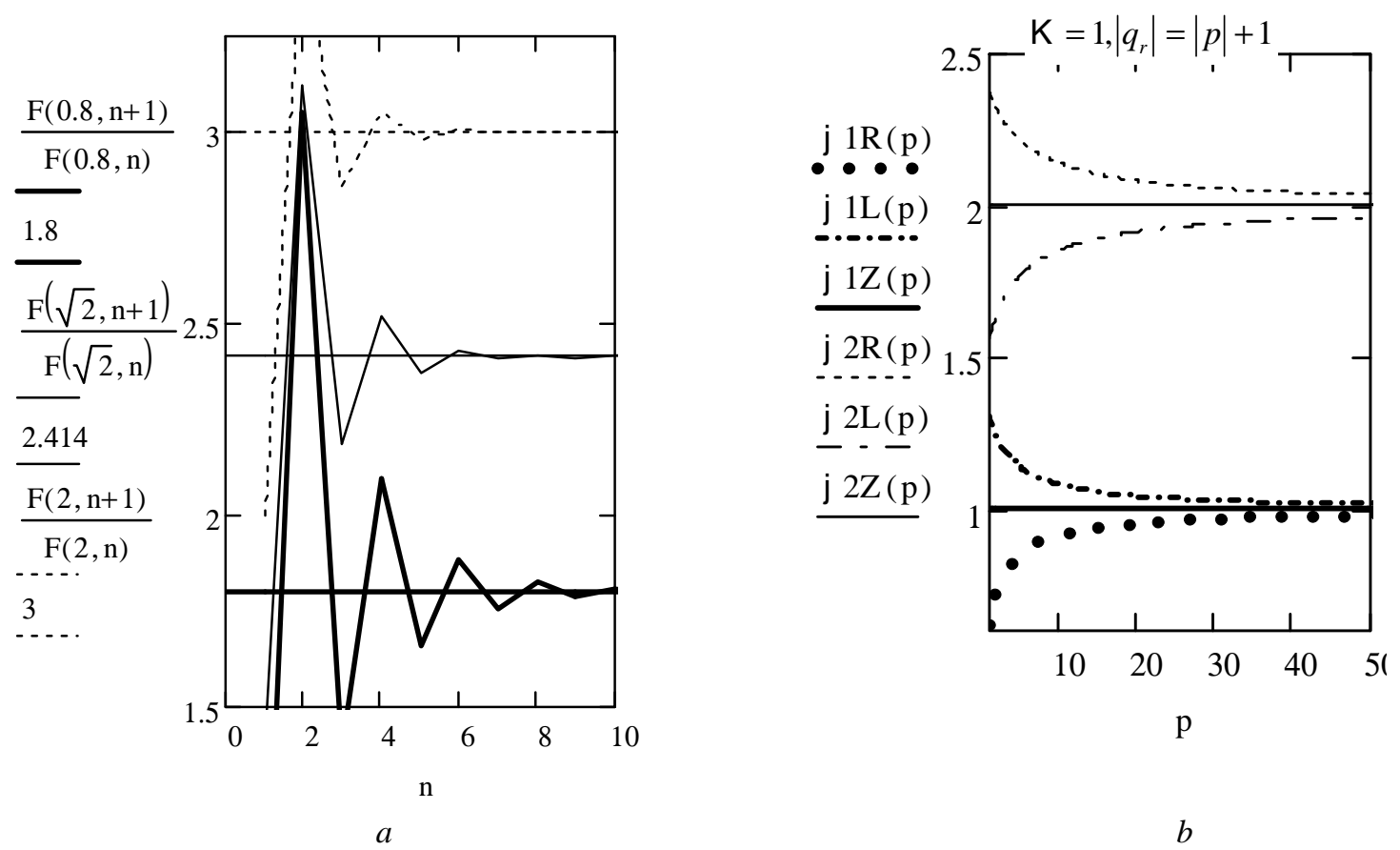

Fig. 2. The oscillation of the ratio $\frac{F(n+1)}{F(n)}$ along the phase direction of the first $\mathrm{K}=1$ root line (a);

The illustration of the boundaries to which the solutions to characteristic equations of the second order recurrence sequence approach asymptotically with the increase of coefficients $p, q$. Here number 1 and 2 according first $\mathrm{K}=1$ and second $\mathrm{K}=2$ root line; indexes $R, L$ according right $\left|q_{r}\right|=|p|$ and left phase satellites of the root line and $Z$ according root line $(b)$

\section{Conclusion}

The study shows that the classical oscillations of the ratio of neighboring members of the Fibonacci sequences are valid for arbitrary directions on the plane of the phase coordinates, approaching, to a maximum, the solutions to the characteristic quadratic equation at a given point. The values of the solutions to the characteristic equation along the satellites are asymptotically close to their integer values of the corresponding root lines.

1. Kosobutskyy P. Modelling of electrodynamic Systems by the Method of Binary Seperation of Additive Parameter in Golden Proportion. Jour. of Electronic Research and Application, 2019,3(3), p. 8-12,

2. Kosobutskyy P. et.al. Physical principles of Optimization of the Static Regime of a Cantilever-Type Powereffect Sensor with a Constant Rectangular Cross Section. Jour. of Electronic Research and Application, 2018, 2(5), p. 11-15.

3. Vorobyov N. Fibonacci Numbers. Moscow, 1961.

4. R. Dunlap. The golden ratio and Fibonacci numbers. World Scientific Publishing Co. Pte. Ltd. 1997

5. Vajda S. (1989) Fibonacci \& Lucas Numbers, and the Golden Section. Theory and Applications. Ellis Horwood limited.

6. Koshy T. (2001) Fibonacci and Lucas numbers with application, A Wiley-Interscience Publication: New York.

7. Horadam A. Basic Properties of a Certain Generalized Sequence of Numbers. Fibonacci Quarterly, 3.3(1965), pp. 161-176.

8. Larcombe P. Horadam Sequences: A Survey Update and Extension, Bulletin of the ICA, Vol. 80 (2017), $99-118$. 
On the universal regularity of the numbers of generalized recurrence sequence and solutions...

9. F. Gatta, A. D'amico. Sequences $\{H n\}$ for which Hn+1/Hn approaches the Golden Ratio. Fibonacci Quarterly, 46/47.4 (2008/2009), pp. 346-349.

10. Ozvatan M., Pashev O. Generalized Fibonacci Sequences and Binnet-Fibonacci Curves. arXiv:1707.09151v1 [math.HO] 28 Jul 2017. https://arxiv.org/pdf/1707.09151.pdf

11. Szakacs T. K-order Linear Recursive Sequences and the Golden Ratio. Fibonacci Quarterly, 55.5 (2017), pp. 186-191.

12. Shneider R. Fibonacci numbers and the golden ratio. VarXiv:1611.07384v1 [math.HO] 22 Nov 2016.

П. С. Кособуцький

Національний університет Львівська політехніка

\section{ПРО ЗАКОНОМІРНОСТІ ФОРМУВАННЯ РЕКУРЕНТНИХ ПОСЛІДОВНОСТЕЙ $\left\{\alpha_{n}\right\}$}

$$
\text { I }\left\{\beta_{n}\right\} \text { В ДЕКОМПОзИцІї } \varphi^{n}=\alpha_{n} \cdot \varphi+\beta_{n}
$$

() Кособучький П., 2019

У роботі досліджено закономірності відношень коефіщієнтів $\alpha_{n}, \beta_{n}$ послідовностей $\left\{\alpha_{n}\right\}$ i $\left\{\beta_{n}\right\}$, які формуються в процесі степеневого перетворення (декомпозиції) виду $\varphi^{n}=\alpha_{n} \cdot \varphi+\beta_{n}$ в ділянці додатних і від'ємних показників $n$.

Ключові слова: пропорція нерівного поділу цілого, декомпозиція, рекурентні послідовності чисел Фібоначчі, формула Біне 